\title{
Integrated molecular, physiological and in silico characterization of two Halomonas isolates from industrial brine
}

\section{Authors: Ross P. Carlson, Olusegun Oshota, Matt Shipman, Justin A. Caserta, Ping Hu, Charles W. Saunders, Jun Xu, Zackary J. Jay, Nancy Reeder, Abigail Richards, Charles Pettigrew, \& Brent M. Peyton}

NOTICE: The final publication is available at Springer via http://dx.doi.org/10.1007/ s00792-015-0806-6.

Carlson RP, Oshota O, Shipman M, Caserta JA, Hu P, Saunders CW, Xu J, Jay ZJ, Reeder N, Richards A, Pettigrew C, Peyton BM, "Integrated molecular, physiological and in silico characterization of two Halomonas isolates from industrial brine," Extremophiles 2016 May 20(3):261-74. 


\title{
Integrated molecular; physiological and in silico characterization of two Halomonas isolates from industrial brine
}

\author{
Ross P. Carlson ${ }^{1,2} \cdot$ Olusegun Oshota ${ }^{1,4} \cdot$ Matt Shipman $^{1,5} \cdot$ Justin A. Caserta ${ }^{3}$. \\ Ping $\mathrm{Hu}^{3}$ - Charles W. Saunders ${ }^{3}$. Jun $\mathrm{Xu}^{3}$ - Zackary J. Jay ${ }^{1,2}$ - Nancy Reeder ${ }^{3}$. \\ Abigail Richards ${ }^{1,2}$. Charles Pettigrew ${ }^{3}$ - Brent M. Peyton ${ }^{1,2}$
}

\author{
Ross P. Carlson \\ ross.carlson@montana.edu \\ Brent M. Peyton \\ bpeyton@montana.edu
}

\begin{abstract}
Two haloalkaliphilic bacteria isolated from industrial brine solutions were characterized via molecu-lar, physiological, and in silico metabolic pathway analy-ses. Genomes from the organisms, designated Halomonas $\mathrm{BC} 1$ and $\mathrm{BC} 2$, were sequenced; $16 \mathrm{~S}$ ribosomal subunit-based phylogenetic analysis revealed a high level of simi-larity to each other and to Halomonas meridiana. Both strains were moderate halophiles with near optimal spe-

cific growth rates $\left(\geq 60 \% \mu_{\max }\right)$ observed over $<0.1-5 \%$ $(\mathrm{w} / \mathrm{v}) \mathrm{NaCl}$ and $\mathrm{pH}$ ranging from 7.4 to 10.2 . Isolate $\mathrm{BC} 1$ was further characterized by measuring uptake or synthesis of compatible solutes under different growth conditions; in complex medium, uptake and accumulation of external glycine betaine was observed while ectoine was synthe-sized de novo in salts medium. Transcriptome analysis of isolate $\mathrm{BC} 1$ grown on glucose or citrate medium measured differences in glycolysis- and gluconeogenesis-based metabolisms, respectively. The annotated $\mathrm{BC} 1$ genome was used to build an in silico, genome-scale stoichiomet-ric metabolic model to study catabolic energy strategies and compatible solute synthesis under gradients of oxygen and nutrient availability. The theoretical analysis identified energy metabolism challenges associated with acclimation to high salinity and high $\mathrm{pH}$. The study documents cen-tral metabolism data for the industrially and scientifically important haloalkaliphile genus Halomonas.
\end{abstract}

Keywords

Halophile - Halomonas - Brine - Compatible solute Systems biology
1 Department of Chemical and Biological Engineering, Montana State University, Bozeman, MT 59717,

2 USA for for Biofilm Engineering, Montana State University, Bozeman, MT 59717, USA

3 Procter and Gamble Co., Cincinnati, OH 45202, USA

4 Present Address: Department of Veterinary Medicine, University of Cambridge, Cambridge, UK

5 Present Address: U.S. Navy, Washington, DC, USA

\section{Introduction}

Halophiles, salt-loving organisms, are highly diverse, widely distributed and include members from all three domains of life (Kushner and Kamekura 1988; Oren 2008). A 'moderate halophile' is defined as an organism that grows optimally at $2.9-14.6 \%(w / v) ~ \mathrm{NaCl}$ while a 'halophile' grows optimally at $\mathrm{NaCl}$ concentrations above $14.6 \%$ (w/v) (Kushner 1978). High salt environments are common yet diverse and include marine systems, seasonal lakes, preserved food, medical wounds and geothermal springs. Life in environments with high salt concentration requires strategies to balance cellular osmotic pressure (Grant 2004; Ventosa et al. 1998). Two distinct osmoregulatory strategies have been described in halophilic bacteria, namely the 'organic-solutes-in' strategy and the 'high-salt-in' strategy (Oren 2008). The organic-solutes-in strategy excludes salt from the cytoplasm while synthesizing or accumulat-ing organic molecules to counter osmotic pressure. These organic solute molecules, known as compatible solutes, 
are often low molecular weight, polar, uncharged or zwitterions (Brown 1976; Grammann et al. 2002; Kempf and Bremer 1998; Kuhlmann et al. 2008). Commonly observed compatible solutes include glycine betaine, ectoine/hydroxyectoine, amino acids, sugars and sugar alcohols (Aston and Peyton 2007; Oren 2008; Roberts 2005). The organicsolutes-in strategy is energetically expensive; it requires pumping ions from the cell and requires the synthesis or import of compatible solutes (Oren 2008). The strategy is observed primarily in microorganisms with high yielding energy metabolisms such as aerobic respiration and phototrophy (Oren 1999, 2011). The high-salt-in strategy accumulates high intracellular concentrations of $\mathrm{K}^{+}$or $\mathrm{Na}^{+}$and requires enzymes to operate near saturating salt concentrations (Grant 2004). This strategy does not necessitate large energy expenditures to pump ions or synthesize compatible solutes, but it does require enzyme amino acid sequences that maintain function at high ionic conditions.

Alkaliphilic organisms grow at high $\mathrm{pH}$; a common definition classifies an organism as an alkaliphile if it grows at $\mathrm{pH}$ values above 10 and has near optimal growth at $\mathrm{pH} 9$ or higher (Goto et al. 2005). Physiological adaptations associated with growth at alkaline $\mathrm{pH}$ include elevated cytosolic $\mathrm{pH}$ with levels reported in the range of 8-9 (Booth 1985; Krulwich et al. 1996). While the internal $\mathrm{pH}$ values are alkaline, they can be up to $2 \mathrm{pH}$ units more acidic than the extracellular environment. $\mathrm{Na}^{+} / \mathrm{H}^{+}$antiporters are critical for maintaining this $\mathrm{pH}$ gradient; intracellular $\mathrm{Na}^{+}$ is exchanged for extracellular $\mathrm{H}^{+}$to acidify the cytosol (Booth 1985). The reliance of alkaliphilic strategies on $\mathrm{Na}^{+}$ to acidify the cytosol is hypothesized to partially explain the co-occurrence of halophilic and alkaliphilic characteristics in some microorganisms (Ventosa et al. 1998).

The genus Halomonas belongs to the Gram-negative family Halomonadeceace. Halomonas strains inhabit environments of $<0.5$ to $\sim 30 \%$ (w/v) salinity and tolerate a wide $\mathrm{pH}$ range (5-10+) (Oren 2008; Ventosa et al. 1998). Halomonas are model haloalkaliphiles studied to understand ecological adaptations to high salinity and high $\mathrm{pH}$ environments. In addition, Halomonas species are of industrial interest because they synthesize the commercial product ectoine/hydroxyectoine, and because they hold promise for polyhydroxyalkanoate synthesis (Guzman et al. 2009; Horikoshi 1999; Mothes et al. 2008; Oren 2011; Pastor et al. 2013; Schwibbert et al. 2011). The current study presents two new, industrial brine Halomonas isolates. The goal of the manuscript is to provide a functional and theoretical characterization of the Halomonas isolates with an emphasis on the metabolic capabilities that make them ecologically competitive in saline and alkaline environments. The study makes substantial contributions to the Halomonas knowledge base by reporting two new genomes, by measuring metabolic acclimations to $\mathrm{pH}$ and salinity stresses and by constructing an in silico, genomescale metabolic reconstruction. The in silico reconstruction provides a systems format for quantifying the physiology, thus establishing a broad rational basis for guiding future research of general and industrial microbiological relevance.

\section{Materials and methods}

\section{Strain isolation and growth conditions}

Halomonas $\mathrm{BC} 1$ and $\mathrm{BC} 2$ were isolated using standard culturing techniques from industrial brine solutions. The strains have been deposited at MicroBioLogics as Halomonas $\mathrm{BC} 1$ and $\mathrm{BC} 2$ (St. Cloud, $\mathrm{MN}$ ). Cultures were grown with either TSB medium or modified M63 (MM63) defined salts medium $\left(15 \mathrm{mM}\left(\mathrm{NH}_{4}\right)_{2} \mathrm{SO}_{4}, 1.8 \mu \mathrm{M} \mathrm{FeSO}{ }_{4}\right.$,

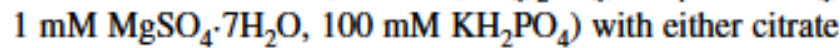
or glucose as the reduced carbon source, at the concentration listed. Both media were augmented with $0-10 \%(w / v)$ $\mathrm{NaCl}$ and had a starting $\mathrm{pH}$ adjusted to 6.7-10.7.

Growth studies were performed in triplicate (three biological replicates/condition) in 96-well plates. Briefly, a starter culture was made for each condition and allowed to grow overnight at $32{ }^{\circ} \mathrm{C}, 150 \mathrm{rpm}$. After $16-20 \mathrm{~h}$ of growth, dilutions were made with appropriate medium so starting culture $A_{600}=\sim 0.01,200 \mu \mathrm{L}$ aliquots of cell suspension in medium were added to each well of a 96 well plate. The plates were sealed with BreathEasy (Diversified Biotech) to allow gas exchange while minimizing evaporation. Six wells per plate were left un-inoculated as controls to monitor evaporation. Plates were run in a kinetics-enabled, temperature controlled $\left(32{ }^{\circ} \mathrm{C}\right)$ plate reader with continuous shaking at $150 \mathrm{rpm}$. Culture absorbance readings were recorded every $5 \mathrm{~min}$ for each well. Cultures were grown and absorbance data $(600 \mathrm{~nm})$ were collected for $14 \mathrm{~h}$. Cultures grown at $<0.1 \%$ (w/v) $\mathrm{NaCl}$ conditions were started in TSB $+0.5 \%$ (w/v) $\mathrm{NaCl}$, and subsequently diluted with TSB containing $0 \%(w / v) ~ N a C l$ prior to the plate growth experiments. Growth was not observed at $0 \%(w / v) ~ N a C l$.

\section{Metabolite analysis}

Complex medium cultures were grown in TSB (without glucose) at $\mathrm{pH} 9$ supplemented with $2.5 \%$ (w/v) $\mathrm{NaCl}$, while minimal medium cultures were grown in MM63 at $\mathrm{pH} 9$ supplemented with $0.35 \%$ (w/v) citrate and $2.5 \%$ (w/v) $\mathrm{NaCl}$. Extracellular and intracellular metabolite analysis was performed by Metabolon, Inc. (Durham, NC, USA). Cell pellet and supernatant fractions were harvested as a function of time. Samples from three separate flasks were analyzed for each time point and were split into equal 
volumes for analysis on the GC/MS and LC/MS/MS platforms (Evans et al. 2009). Metabolites were identified by matching the ions' chromatographic retention index and mass spectral fragmentation signatures to the Metabolon standards library. Relative metabolite concentration quantification used peak area integration. Analysis provided metabolite concentration trends, but not absolute concentrations. The method did not permit analysis of small volatile molecules like acetate or ethanol.

\section{Genome sequencing and assembly}

The genomes were sequenced using a whole genome shotgun strategy. Briefly, cultures were grown in rich TSB $(50 \mathrm{~g} / \mathrm{L})$ at $\mathrm{pH} 9$ supplemented with $2.5 \%$ (w/v) $\mathrm{NaCl}$ and $0.1 \%(\mathrm{w} / \mathrm{v})$ sodium borate. Cells were harvested after $16 \mathrm{~h}$ of batch growth (older culture samples plugged separation columns) and DNA was purified in accordance with Qiagen genomic DNA procedures. $20 \mu \mathrm{g}$ of purified, genomic DNA from Halomonas $\mathrm{BC} 1$ and $\mathrm{BC} 2$ were sent to Agencourt Bioscience, Inc. (Beverly, MA USA) for sequencing. $3 \mathrm{~kb}$ mate paired libraries for the $\mathrm{BC} 1$ and $\mathrm{BC} 2$ genomes were constructed. Sequencing fragments were detected via the Genome Sequencer Roche 454 Titanium System ${ }^{\mathrm{TM}}$, which provided $20.8 \times$ and $16.2 \times$ coverage for $\mathrm{BC} 1$ and $\mathrm{BC} 2$, respectively. Reads were assembled into contigs and super contigs by Newbler assembler; fasta files and associated quality scores files were generated for the consensus. Assembly resulted in 20 scaffolds for $\mathrm{BC} 1$ and 29 scaffolds for $\mathrm{BC} 2$. The 16S rRNA gene was sequenced separately for each isolate. Genome sequences for $\mathrm{BC} 1$ and $\mathrm{BC} 2$ are available at NCBI as GDsub28059 and GDsub28060 respectively.

\section{ORF prediction and annotation}

Open reading frame (ORF) coding sequences and peptide sequences were predicted using Glimmer v3 (Delcher et al. 2007); GeneMark (Georgia Institute of Technology, Atlanta, GA) was used to verify the coding frame and identify the missing reading frames. The genomes of $\mathrm{BC} 1$ and $\mathrm{BC} 2$ were annotated putatively using RAST (Argonne National Laboratory, IL, USA).

\section{Phylogenetic analyses of Halomonas BC1 and BC2}

The complete BC1 16S rRNA gene (1,522 nt) was obtained from the genome sequence while the BC2 16S rRNA gene (1,361 nt) was determined with Sanger sequencing of PCR products generated with universal bacterial primers (the assembled $\mathrm{BC} 2$ genome sequence did not contain a complete 16S rRNA gene). Sequence alignments were generated using ClustalW (Thompson et al. 1994), manually edited, and then used to construct an unrooted phylogenetic tree using the maximum likelihood method; bootstrap values were determined with 10,000 resamplings using MEGA version 6.06 (Tamura et al. 2013). Reference 16S rRNA gene sequences were obtained from either NCBI GenBank or the DOE-Joint Genome Institute Integrated Microbial Genomes (JGI-IMG) database using blastn.

\section{DNA microarray preparation, transcriptome and data analysis}

Affymetrix gene chips were designed using unique coding frames from the Halomonas $\mathrm{BC} 1$ and $\mathrm{BC} 2$ genomes. The custom arrays were synthesized by Affymetrix, Inc. (USA) and consisted of 9,790 probes; 8-11 probe pairs were selected per sequence and probe selection region involved 600 bases from the $3^{\prime}$ gene end.

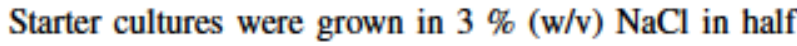
strength TSB at $\mathrm{pH} 8.5$ for $18 \mathrm{~h}$ at $32{ }^{\circ} \mathrm{C}$ with shaking. Starter cultures were washed with $0.9 \%$ (w/v) $\mathrm{NaCl}$ and inoculated 1:50 into MM63 medium containing either $0.4 \%$ (w/v) glucose or $0.4 \%(w / v)$ citrate as the carbon source. Cultures were then incubated for $18 \mathrm{~h}$ at $32^{\circ} \mathrm{C}$ with shaking. Four replicates of each growth condition were performed.

After incubation, RNA was isolated using Qiagen RNeasy Mini Kit according to the manufacturer's instructions. $4 \mathrm{~mL}$ of each culture was centrifuged for $10 \mathrm{~min}$ at $3500 \mathrm{rpm}$ to pellet cells. Cell pellets were washed with $1 \mathrm{~mL}$ of cold PBS $+2 \mathrm{~mL}$ of RNAprotect Bacterial Reagent (Qiagen). Cells were pelleted by centrifugation at $3500 \mathrm{rpm}$ for $10 \mathrm{~min}$. After removing the supernatant, $200 \mu \mathrm{L}$ of lysozyme ( $15 \mathrm{mg} / \mathrm{mL}$ in TE) was added to each pellet and resuspended by pipetting and vortexing. Resuspended pellets were incubated for $10 \mathrm{~min}$ at room temperature with $10 \mathrm{~s}$ vortexing every $2 \mathrm{~min}$ of incubation. Following incubation, $700 \mu \mathrm{L}$ of Buffer RLT was added and tubes were vortexed, followed by the addition of $500 \mu \mathrm{L}$ of $100 \%$ ethanol. Tubes were inverted to mix. This lysate was added to an RNeasy column and spun for $30 \mathrm{~s}$ at $10,000 \times \mathrm{g}$. Qiagen DNase solution ( $80 \mu \mathrm{L}$ ) was added to each column and incubated for $15 \mathrm{~min}$ at room temperature. Columns were washed by adding $350 \mu \mathrm{L}$ of Buffer RW1 and centrifuging for $30 \mathrm{~s}$ at $10,000 \times g$. The columns were then washed twice with $500 \mu \mathrm{L}$ of Buffer RPE. $30 \mu \mathrm{L}$ of prewarmed $\left(37^{\circ} \mathrm{C}\right)$ RNase-free $\mathrm{H}_{2} \mathrm{O}$ was added to each column and incubated at room temperature for $3 \mathrm{~min}$. After incubation, tubes were centrifuged for $1 \mathrm{~min}$ at $10,000 \times \mathrm{g}$ to collect isolated RNA. RNA concentration was measured using a Nanodrop (Thermo Scientific).

$500 \mathrm{ng}$ of purified total RNA was converted to cRNA GeneChip target using the Ambion (Austin, TX) MessageAmp II Bacteria RNA Amplification kit and protocol 
provided. $15 \mu \mathrm{g}$ each of GeneChip targets were hybridized overnight to the custom Affymetrix Halomonas GeneChips. Following hybridization, GeneChips were washed and stained using the protocol provided by Affymetrix and scanned using the Affymetrix GeneChip Scanner 3000 with Autoloader. Image data was summarized using the Affymetrix MAS5.0 algorithm followed by statistical QC analysis to confirm data quality.

Statistical analysis of differential transcript expression was performed using the Limma Bioconductor package in R (Smyth 2005; http://www.bioconductor.org). Briefly, the data were normalized in Bioconductor using the quantile normalization function in the preprocess Core package. Signal data were then analyzed using the Limma Bioconductor package. The Limma method employs linear models and empirical Bayes methods (Smyth 2004) for assessing differential expression in microarray experiments. With this approach, estimates of the fold changes and standard errors were obtained by fitting a linear model to the log-expression data for each gene from experiments. Empirical Bayes smoothing was then applied to the standard errors to derive moderated $t$-statistics, moderated $F$-statistics, and log-odds of differential expression. Probability values were adjusted by the Benjamini-Hochberg method (Benjamini and Hochberg 1995). Differential gene expression was referred to as significant, if the adjusted $p$ value was $\leq 0.05$ and the $\log _{2}$ fold change was $\geq 1.5$ or $\leq 1.5$.

\section{Construction of Halomonas metabolic network model}

A Halomonas BC1 stoichiometric metabolic network model was constructed using the annotated genome (supplementary material), transcriptomic studies and a literature review (Ates et al. 2011; Schwibbert et al. 2011). The model documents 620 genes which were used to define 277 reactions some of which represent a manually compressed sequence of enzymes such as sequences associated with linear biosynthetic pathways. The Halomonas BC2 genome was used to fill gaps in the metabolic model when an appropriate Halomonas $\mathrm{BC} 1$ gene could not be identified (supplementary material). The in silico model reconstructs the central metabolism with the associated anabolic and catabolic biochemical pathways for biomass synthesis, maintenance energy generation and compatible solute synthesis. The model does not include macromolecular degradation pathways to avoid excessive futile cycling. Simulations considered minimal medium cultivation with either glucose or citrate as the sole reduced carbon and energy source and $\mathrm{NH}_{4}{ }^{+}$as the sole nitrogen source. Common byproducts of Halomonas metabolism were included (e.g. acetate, formate, lactate, succinate, ethanol, acetoin).

The biomass synthesis reaction considered the six major cellular macromolecules: protein, DNA, RNA, phospholipids, lipopolysaccharide, and peptidoglycan, as well as ectoine, putrescine and ash (supplementary material). The protein amino acid composition was derived from the codon usage frequency in the genomic sequence of Halomonas $\mathrm{BC} 1$. The composition of DNA was calculated based on G + C content of the genomic sequence of Halomonas $\mathrm{BC} 1$. As the predominant RNA is rRNA, nucleotide composition of rRNA was calculated from the 16S rRNA sequence and used as the representative composition for the RNA macromolecule reaction. The phospholipid reaction equation was derived from published Halomonas data (Giordano et al. 2007). Biomass macromolecular mass fractions were based on previous studies of similar bacteria (Ates et al. 2011; Neidhardt et al. 1990; Schwibbert et al. 2011). The biomass synthesis reaction includes ATP for polymerization, but does not include the separate maintenance energy requirements. The in silico model with reaction stoichiometry, genome basis for each reaction, biomass construction files and atomic balancing files are all provided in the supplemental material.

\section{Computation of elementary flux modes (EFMs)}

The in silico metabolic model was analyzed using elementary flux mode analysis (EFMA). Briefly, elementary flux modes (EFMs) are mathematically defined, minimal, nondivisible metabolic pathways. Each EFM is comprised of a unique combination of biochemical steps (Schuster and Schuster 1993; Schuster et al. 2000; Schuster and Hilgetag 1994). EFMs can be used to represent all steady state phenotypes in the model-defined physiological flux space through nonnegative linear combinations (Trinh et al. 2009). EFMs were enumerated using CellNetAnalyzer (Klamt et al. 2007) and analysed using MATLAB or MS Excel.

\section{Results}

\section{Genome and phylogenetic analysis}

Isolate $\mathrm{BC} 1$ and $\mathrm{BC} 2$ genomes were sequenced and analyzed for gene content. The $\mathrm{BC} 1$ and $\mathrm{BC} 2$ genomes consisted of one putative chromosome of $3,708,838$ and $3,400,070 \mathrm{bp}$, respectively, with similar GC content (59.9 and $60.1 \%$ ) (Table 1). The protein-coding densities for the two genomes were similar (BC1: $79.5 \%$; BC2: $79.9 \%$ ), but the number of protein-encoding genes predicted for BC2 $(3,194)$ was higher than for BC1 $(2,954)$ (Table 1). $\mathrm{BC} 1$ and $\mathrm{BC} 2$ genomes encoded 59 RNA genes comprising 56 tRNA genes and one copy of the rRNA operon (16S rRNA and 23 rRNA, 5S rRNA sequences were not identified but were assumed present). RAST ORF (open reading 
Table 1 Summary of Halomonas $\mathrm{BC} 1$ and $\mathrm{BC} 2$ genomes

\begin{tabular}{lll}
\hline & BC1 & BC2 \\
\hline Number of chromosomes & 1 & 1 \\
Chromosomal size & $3,708,838$ bp & $3,400,070 \mathrm{bp}$ \\
No. of scaffolds & 20 & 29 \\
G + C content & $59.9 \%$ & $60.1 \%$ \\
Genes, total number & 3013 & 3253 \\
Coding density & $79.5 \%$ & $79.9 \%$ \\
Protein coding genes & 2954 & 3194 \\
Total RNA genes & 59 & 59 \\
5S rRNA & $1^{\mathrm{a}}$ & $1^{\mathrm{a}}$ \\
16S rRNA & 1 & 1 \\
23S rRNA & 1 & 1 \\
tRNA genes & 56 & 56 \\
\hline
\end{tabular}

a $5 \mathrm{~S}$ rRNA sequence was not identified, its presence was assumed

frame, mcs.anl.gov) analysis of the genomes is provided in the supplemental material.

Phylogenetic analysis of the BC1 and BC2 16S rRNA genes established the taxonomic assignment of these organisms in the Halomonas genus and revealed that these sequences were most closely related to each other and to $H$. meridiana S1 and to H. stevensii S18214 (Fig. 1). The BC1 and BC2 16S rRNA genes shared $99.8 \%$ nucleotide identity to each other. The results indicate that the 16S rRNA gene sequences across these Halomonas microorganisms are highly conserved.
Whole genome sequence comparison was performed between $\mathrm{BC} 1$ and $\mathrm{BC} 2$, and 12 publically available Halomonas genome sequences. $\mathrm{BC} 1$ and $\mathrm{BC} 2$ were more similar to each other than the other sequenced Halomonas species. There was an $80 \%$ sequence identity between the $\mathrm{BC} 2$ genome and the $\mathrm{BC} 1$ genome. Halomonas stevensii was the most-closely related, sequenced Halomonas species, and had a $79.5 \%$ sequence identity with the BC1 genome. A comparison of genome statistics including genome size, putative gene number, $\mathrm{G}+\mathrm{C}$ percentage, and percent sequence identity is presented in the supplemental material due to the large amount of data.

\section{Genome-based metabolic analysis}

The $\mathrm{BC} 1$ and $\mathrm{BC} 2$ genomes encoded a suite of catabolic genes associated with a wide variety of carbohydrates, peptides and organic acids. The $\mathrm{BC} 1$ genome had 11 annotated genes for sugar transport, 36 annotated genes for di- and tri-carboxylic acid transport, 29 genes associated with amino acid or peptide transport as well as an array of transporters and permeases for small organic molecules. The isolate genomes had genes for the complete Embden-Meyerhof-Parnas and Entner-Doudoroff glycolytic pathways, the decarboxylating pentose phosphate pathway and the oxidative tricarboxylic acid (TCA) cycle. The glyoxylate shunt, glycolate dehydrogenase, formate dehydrogenase and acetyl-CoA synthetase genes enable, hypothetically, catabolism of small organic acids.
Fig. 1 Phylogenetic analysis of Halomonas 16S rRNA gene sequences. The tree was constructed using maximum likelihood analysis and bootstrap values were determine by resampling 10,000 replicate trees. Sequences were obtained from either NCBI Genbank or JGI-IMG Genomes (asterisk) and the respective accession number or gene ID is provided in parentheses, respectively. $\mathrm{BC} 1$ and $\mathrm{BC} 216 \mathrm{~S}$ rRNA sequences can be found in the supplemental material (BCl genome and $\mathrm{BC} 2$ genome worksheets respectively) and have been submitted to NCBI

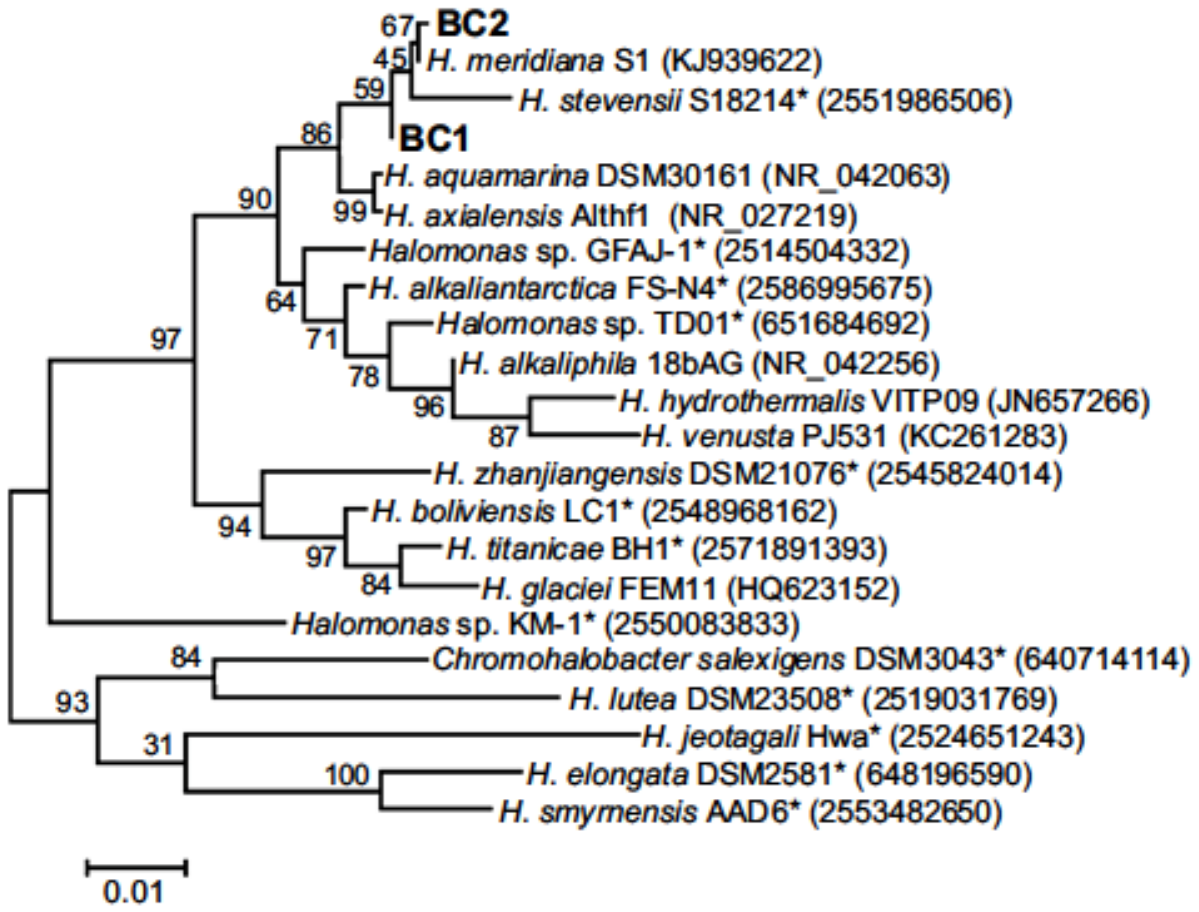


The $\mathrm{BC} 1$ and $\mathrm{BC} 2$ genomes contained a large number of genes associated with the electron transport chain and respiration. Genes for the soluble as well as the membrane-associated transhydrogenase were identified and two NADH oxidoreductases associated with the electron transport chain including a $\mathrm{H}^{+}$motive force (pmf) generating complex were identified. The genomes contained two cytochrome terminal oxidases for oxygen, a dissimilatory and assimilatory nitrate reductase system and a tetrathiosulfate reductase system. The genomes also possessed an rnf operon which links the electron transport chain and the oxidation/reduction of ferredoxin with a $\mathrm{H}^{+}$ motive force (Biegel et al. 2011). The $\mathrm{H}^{+}$motive force could be used with the present $\mathrm{F}_{0} \mathrm{~F}_{1}$-type ATP synthase to generate ATP.

Maintenance of intracellular osmotic pressure, ionic charge and $\mathrm{pH}$ is critical for haloalkaliphiles. Genes were found for numerous transporters involved with physiological ions including $\mathrm{Na}^{+}, \mathrm{H}^{+}, \mathrm{Ca}^{2+}, \mathrm{K}^{+}$and $\mathrm{Cl}^{-}$. $\mathrm{BC} 1$ had 9 genes annotated with $\mathrm{Na}^{+} / \mathrm{H}^{+}$antiporter functionality (Booth 1985). Both genomes also had the $\mathrm{Na}^{+}$-translocating NADH-quinone reductase operon suggesting energy from the electron transport chain could power the translocation of $\mathrm{Na}^{+}$(Steuber et al. 2000; Verkhovsky and Bogachev 2010). The isolate genomes possessed the $\mathrm{Na}^{+}$ pumping oxaloacetate decarboxylase as yet another active $\mathrm{Na}^{+}$transport strategy (Dimroth et al. 2001).

The $\mathrm{BC} 1$ and $\mathrm{BC} 2$ genomes had annotated genes for the de novo ectoine/hydroxyectoine synthesis pathway (Bursy et al. 2007; Schwibbert et al. 2011). Both genomes lacked the genes for de novo glycine betaine synthesis but contained an $\mathrm{ABC}$ proline/glycine betaine transporter gene and a high affinity choline transporter gene. In the presence of extracellular choline, the isolates could hypothetically synthesize glycine betaine using the choline dehydrogenase and glycine betaine dehydrogenase genes. Synthesis pathways for other metabolites with compatible solute-associated function including glutamate, glutamine, proline and glycerol were identified.

The Halomonas isolate genomes had genes associated with storage polymer synthesis and degradation providing a buffer of anabolic resources, cellular energy and reducing equivalents during unbalanced growth. Notable examples include polyhydroxyalkanoate (PHA) synthesis which was supported in the $\mathrm{BC} 1$ genome by three putative PHA synthase genes, two acetoacetyl-CoA reductase genes and five acetyl-CoA acetyltransferase genes as well as a PHA depolymerase gene and two genes for PHA oxidation. A similar gene distribution was present in BC2. Both $\mathrm{BC} 1$ and $\mathrm{BC} 2$ had biosynthesis and degradation genes for the energy and phosphorus storage polymer polyphosphate. The isolates also had polyamine (putrescine) synthesis genes as a reduced nitrogen storage strategy.
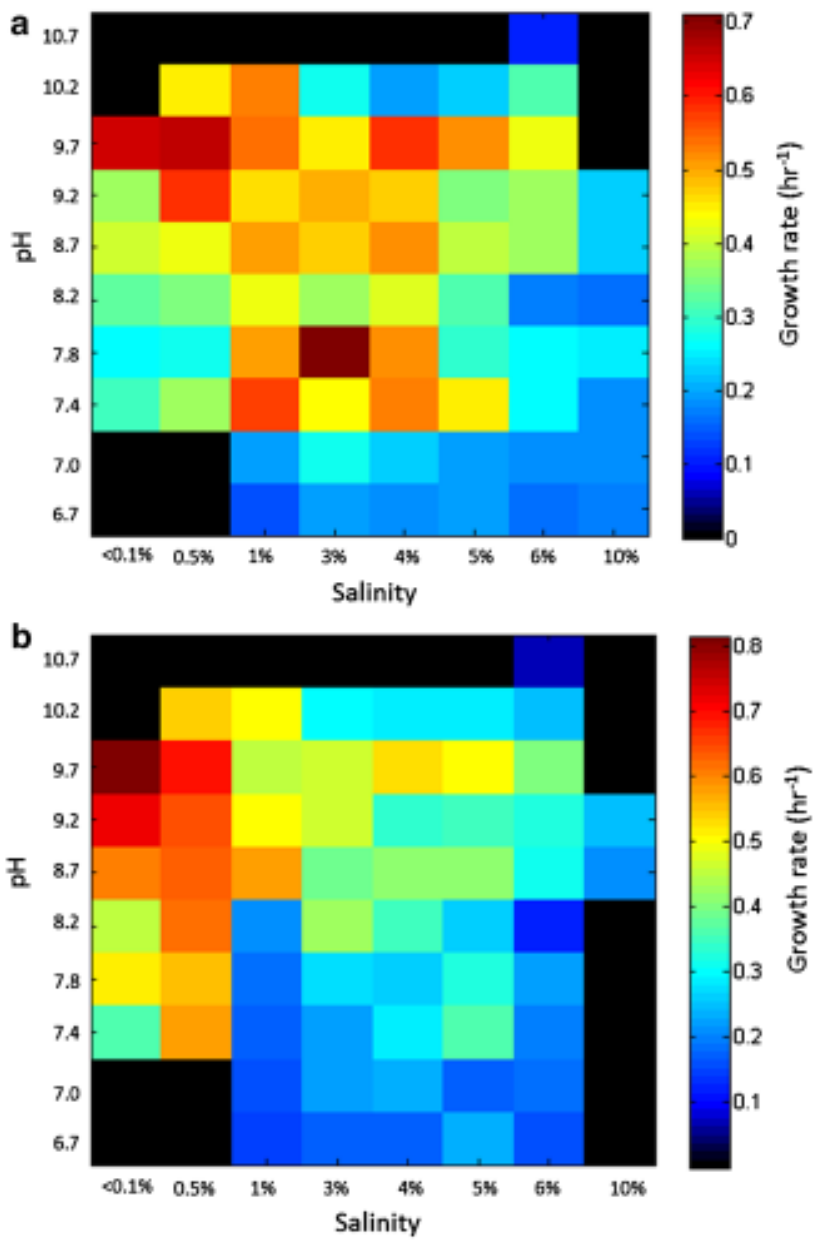

Fig. 2 Heat maps of specific growth rates for Halomonas isolates cultured in TSB medium at $32{ }^{\circ} \mathrm{C}$ as a function of culture salinity $(\mathrm{w} / \mathrm{v} \mathrm{NaCl})$ and $\mathrm{pH}$. Color bar at right denotes the range of specific growth rates $\left(\mathrm{h}^{-1}\right)$. Black sections denote no observed growth. a Halomonas isolate $\mathrm{BC} 1$. b Halomonas isolate $\mathrm{BC} 2$. Each specific growth rate was determined from three independent biological replicates. Additional information including standard deviations of reported values is available in supplemental material

\section{Tolerance to $\mathrm{pH}$ and salinity}

$\mathrm{BC} 1$ and $\mathrm{BC} 2$ tolerance to salinity and $\mathrm{pH}$ was measured during batch growth using a matrix of 80 different medium conditions (salinity $<0.1-10 \%$ (w/v) $\mathrm{NaCl}, \mathrm{pH} 6.7-10.7$ ). Each condition was tested in triplicate with each isolate. $\mathrm{BC} 1$ tolerated a wider range of salinity and $\mathrm{pH}$ than $\mathrm{BC} 2$ although $\mathrm{BC} 1$ had a lower maximum specific growth rate $\left(0.71 \pm 0.1 \mathrm{~h}^{-1}\right)$ than $\mathrm{BC} 2\left(0.82 \pm 0.05 \mathrm{~h}^{-1}\right)$. Isolate $\mathrm{BC} 1$ grew fastest at $3 \%(\mathrm{w} / \mathrm{v}) \mathrm{NaCl}$ and $\mathrm{pH} 7.8$ (Fig. 2a), while $\mathrm{BC} 2$ grew fastest at $<0.1 \%(\mathrm{w} / \mathrm{v}) \mathrm{NaCl}$ and $\mathrm{pH} 9.7$ (Fig. 2b). Culturing conditions within the salinity range of $<0.1-6 \%(\mathrm{w} / \mathrm{v}) \mathrm{NaCl}$ and $\mathrm{pH}$ range of 7.4-10.2 supported $\mathrm{BC} 1$ growth at a specific growth rate $\geq 60 \%$ of $\mu_{\max }$, while culturing conditions within the salinity range of $<0.1-5 \%$ 
(w/v) $\mathrm{NaCl}$ and $\mathrm{pH}$ range of 7.4-10.2 supported $\mathrm{BC} 2$ grow at $\geq 60 \%$ of $\mu_{\max }$. Both BC1 and BC2 had measurable growth at $10 \%(\mathrm{w} / \mathrm{v})$ salinity when the $\mathrm{pH}$ ranged from 8.7 to 9.2 and both had measurable growth at $\mathrm{pH} 10.7$ with a salinity of $6 \%(w / v)$.

\section{Metabolite analysis}

BC1 uptake or synthesis of compatible solutes was measured using mass spectrometry(MS)-based metabolite analysis of both the extracellular supernatant and the cell pellet. Cultures were grown on either complex TSB medium $(2.5 \%(\mathrm{w} / \mathrm{v}) \mathrm{NaCl}, \mathrm{pH}$ 9) or citrate salts medium $(2.5 \%$ $(w / v) ~ N a C l, p H ~ 9)$. The different media resulted in different specific growth rates, so a sampling scheme was applied to compare the cultures at equivalent growth phases labeled time points 1-4. The MS-based analytical methodologies measured qualitative metabolite concentration trends not absolute concentrations.

Relatively high specific intracellular concentrations (normalized to culture DNA) of glycine betaine were measured when $\mathrm{BC} 1$ was grown on TSB as compared to minimal medium (Fig. 3a). Intracellular accumulation of glycine betaine for the TSB grown cultures coincided with a slight decrease in extracellular levels suggesting cellular uptake of metabolite originating in the medium (Fig. 3a, b). Alternatively, $\mathrm{BC} 1$ synthesized higher specific concentrations of ectoine when grown in minimal medium as compared to growth in TSB medium and secreted or leaked ectoine into the medium at the later time points (Fig. 3c, d). Hydroxyectoine was not present in the MS metabolite standards library and therefore could not be analyzed. Extracellular glutamate and proline concentrations decreased during growth in TSB medium but not glutamine (Fig. 3g, i, k). Extracellular concentrations of 3-hydroxybutyrate (3-HB) increased with time during growth in TSB (Fig. 3f). 3-HB is sometimes secreted or leaked from cells accumulating PHB which is both a carbon and electron storage polymer (Carlson et al. 2005) and can also be a byproduct of amino acid or fatty acid catabolism. The analytical technique only detected soluble molecules so it was not possible to measure the insoluble storage polymer PHB.

\section{Transcript analysis}

Coding region transcriptomic data revealed differentially expressed genes during $\mathrm{BC} 1$ batch growth in either glucose $(0.4 \%$ w/v) salts medium or citrate $(0.4 \%$ w/v) salts medium $(\mathrm{pH} 8.5,3 \% \mathrm{NaCl}(\mathrm{w} / \mathrm{v}))$. A total of 532 gene transcripts had an adjusted $p$ value of 0.05 or less suggesting significant expression level differences between the two conditions. Figure $4 a$ depicts the top 43 up-regulated genes ( $\log _{2}$ fold change, adjusted $p$ value $\leq 0.05$ ) in glucose medium relative to citrate medium. Similarly, Fig. 4b shows the top 43 up-regulated genes ( $\log _{2}$ fold change, adjusted $p$ value $\leq 0.05$ ) in citrate medium relative to glucose medium. Figure $4 \mathrm{c}$ summarizes significant differences in central metabolism transcript levels between growth on glucose and citrate. Glucose-grown cultures had increased expression of glycolysis transcripts as well as transcripts associated with an overflow/fermentation metabolism like alcohol and acetaldehyde dehydrogenase while TCA cycle gene transcripts were down regulated. Oxygen cytochromes were also up expressed. The hydroxyectoine synthesis gene ectD, which catalyzes the oxygen dependent synthesis of hydroxyectoine from ectoine, had increased expression during growth on glucose. This may have been a response to high concentrations of overflow metabolites contributing to elevated osmotic stress or it may have been a response to low oxygen concentrations where the elevated enzyme levels would ensure a flux of oxygen for baseline hydroxyectoine synthesis. The citrate-grown culture had up regulated TCA cycle gene transcripts; these genes would be required to catabolize the carbon source for ATP as well as provide the metabolic intermediates for biomass synthesis. The citrate-grown cultures also had up expressed gluconeogenesis gene transcripts including PEP carboxykinase and PEP synthase.

\section{In silico pathway analysis of cellular energy generation and compatible solute synthesis}

Elementary flux mode analysis (EFMA) quantified a measure of the Halomonas $\mathrm{BC} 1$ central metabolism robustness, the number of mathematically distinct metabolic pathways possible for maintenance energy and compatible solute synthesis. For instance, EFMA identified 27,651 and 43,155 mathematically distinct metabolic pathways for producing the amino acid and compatible solute L-glutamate from citrate and glucose, respectively (Table 2 ).

High salinities and high $\mathrm{pH}$ reduce the solubility of some cellular resources like oxygen and iron (Pade et al. 2013). The BC1 in silico metabolic model was used to quantify physiological ramifications of limited resources on compatible solute synthesis. Briefly, each compatible solute synthesizing EFM had its electron donor and electron acceptor requirements calculated (Carlson 2007, 2009). The resource requirements for each EFM were plotted in a two dimensional phenotype space; the biologically feasible subspace was represented by the shaded areas in Fig. 5. The EFMs that minimize the requirement of the two resources define a cost-benefit tradeoff curve (dotted line in Fig. 5a) that quantifies the optimal use of the two resources to produce the compatible solute (Carlson and Taffs 2010). The tradeoff curve is analogous to a Pareto front used in economic analyses (Beck et al. 2016). 
Fig. 3 Metabolomic analysis of relative intracellular (a, c, e, g, $\mathbf{i , ~ k )}$ and extracellular (b, d, f, h, j, l) concentrations of glycine betaine, ectoine, 3-hydroxybutyrate, glutamate, glutamine and proline for Halomonas $\mathrm{BCl}$ cultures grown on citrate salts medium (open circles) or complex TSB medium (filled circles) at $\mathrm{pH} 9$ and salinity $2.5 \%(w / v) ~ N a C l$. Relative intracellular concentrations are on a per culture DNA concentration basis
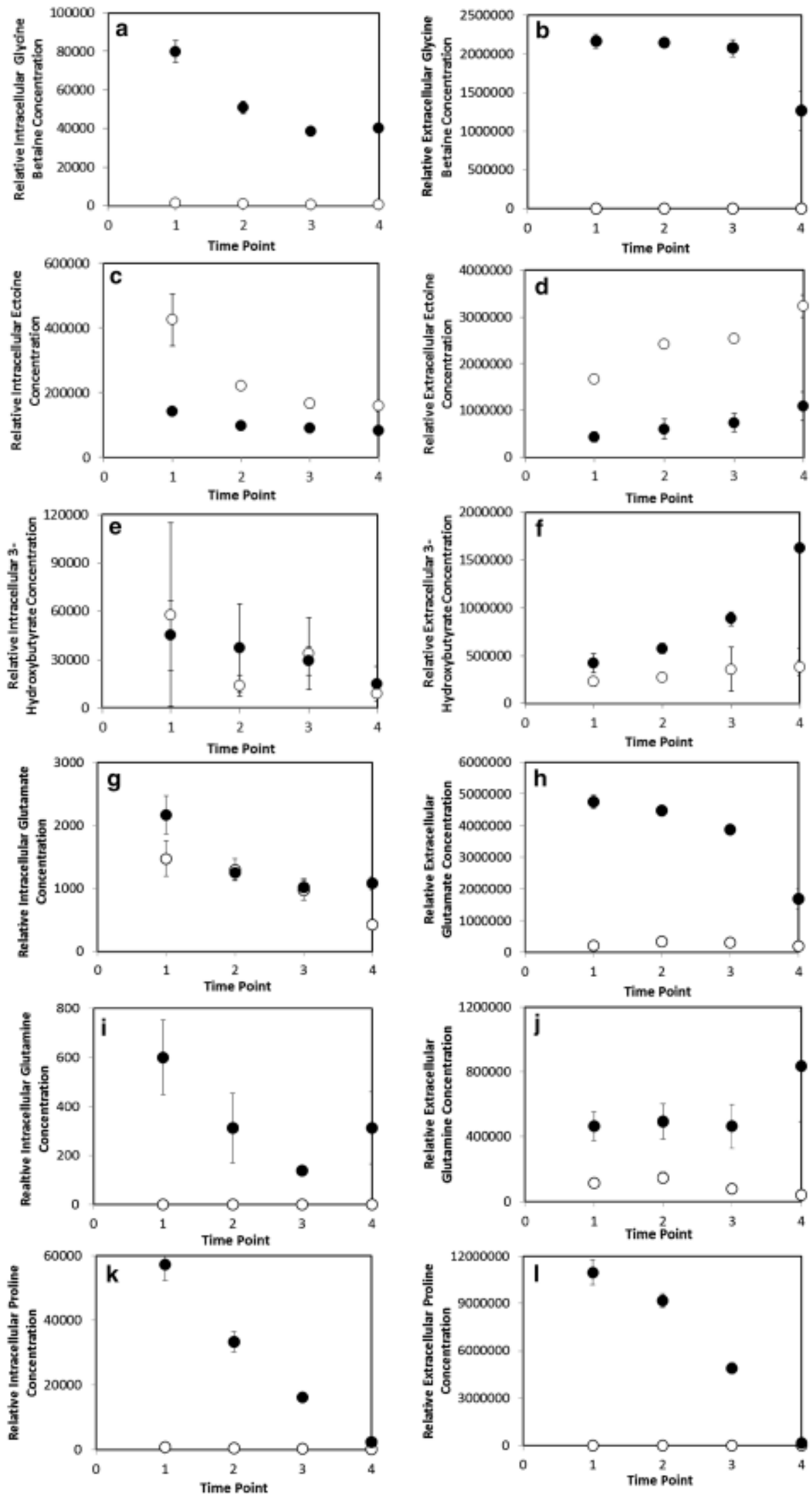
a

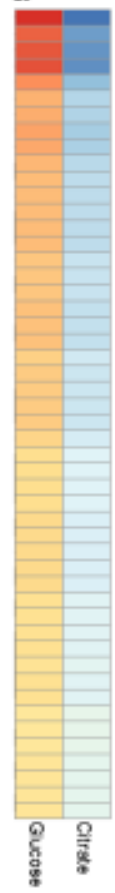

b

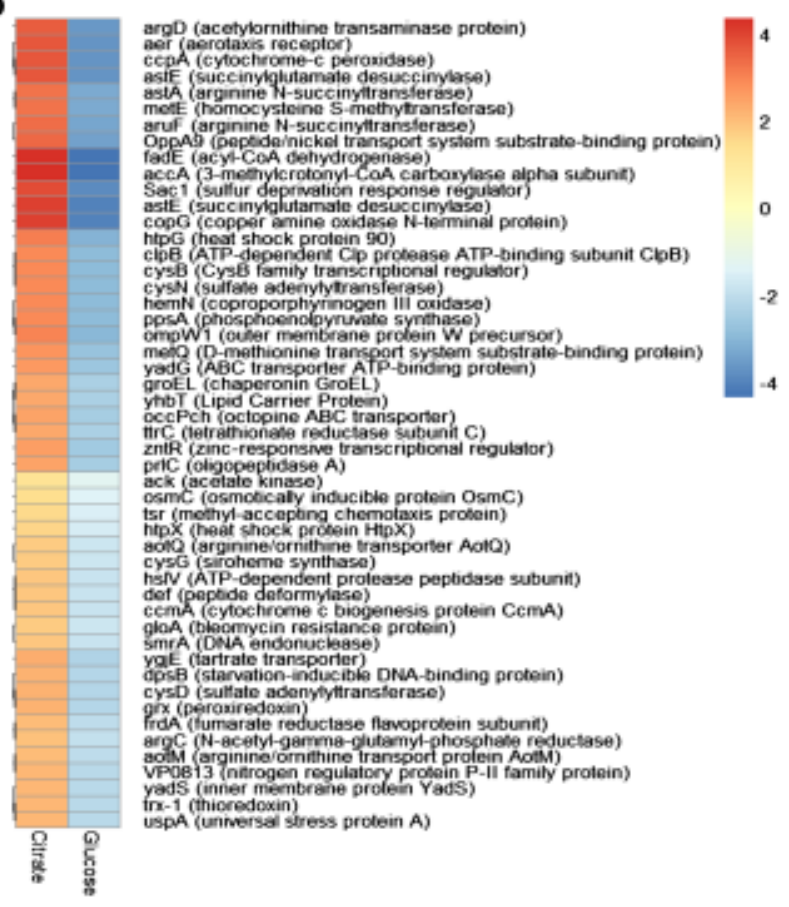

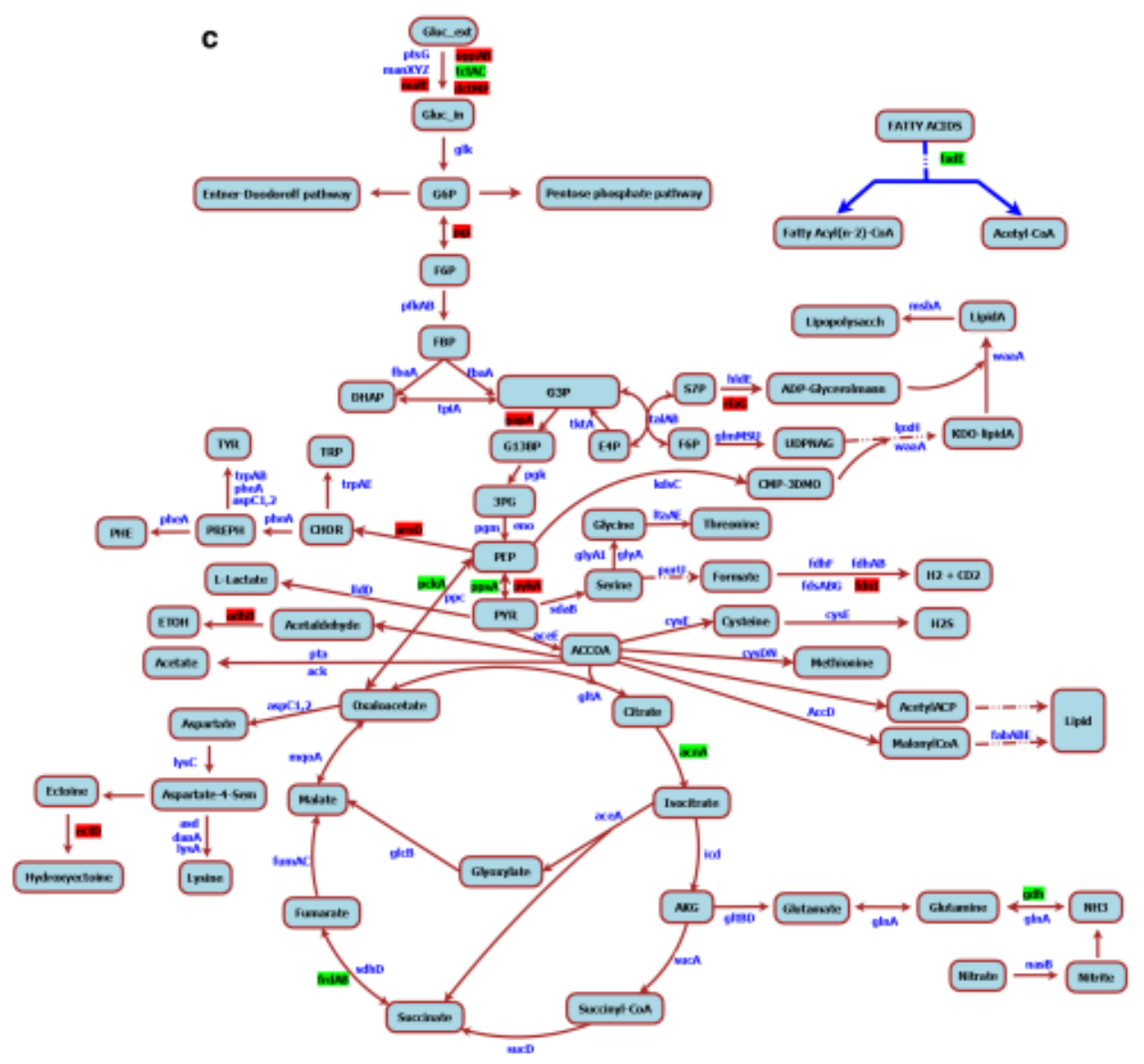

Fig. 4 Halomonas BC1 differential gene expression during growth on glucose and citrate salts media ( $\mathrm{pH} 8.5,3 \%$ (w/v) NaCl). a Top up-expressed genes ( $\log _{2}$ fold) in glucose medium relative to citrate. b Top up-expressed genes in citrate medium relative to glucose. c Diagram illustrating up and down transcript levels mapped to the central metabolism. Genes up-expressed during growth on glucose medium are highlighted in red, and genes up-expressed during growth on citrate are in green, genes with unchanged expression levels are in blue font. All reported genes had a Benjamini-Hochberg adjusted $p$ value $\leq 0.05$ and $\geq 1.5$ or $\leq 1.5 \log _{2}$ fold expression change 


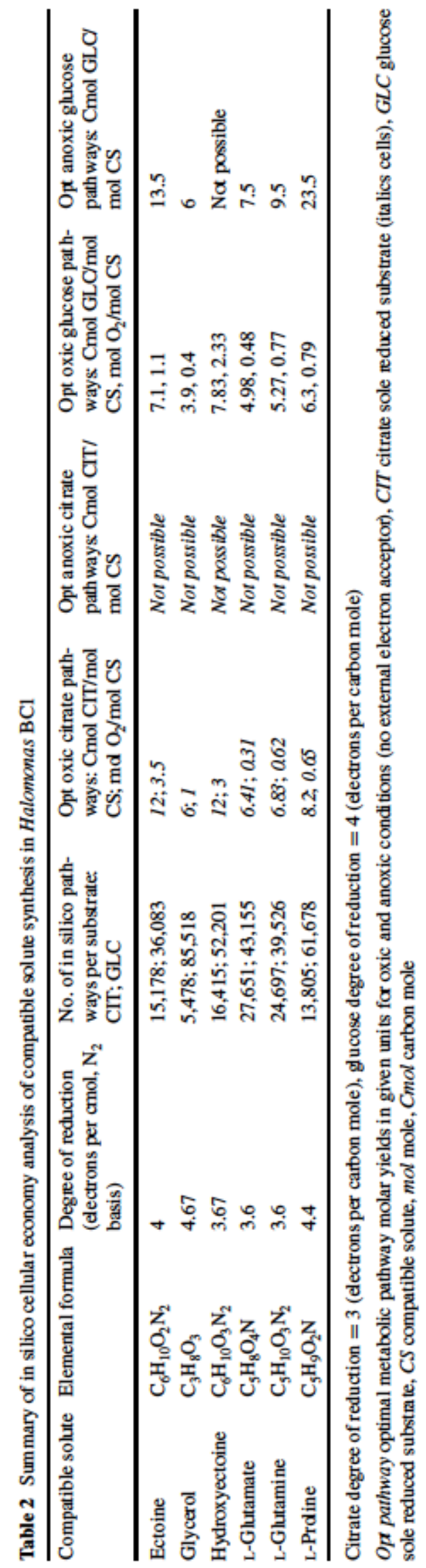

The cost-benefit analyses provided a systems basis for analyzing possible Halomonas acclimations to high salinity while experiencing nutrient scarcity. Figure $5 \mathrm{~b}$, c plot the oxygen and electron donor (glucose, citrate respectively) requirements to synthesize six different compatible solutes (glycerol, glutamate, glutamine, proline, ectoine, hydroxyectoine). The electron donor and electron acceptor requirements to synthesize each compatible solute were a function of the size of the metabolite, the degree of reduction of substrate and compatible solute as well as the structure of the biochemical network (Table 2). Glycerol was the least expensive compatible solute to synthesize in terms of carbon moles ( $\mathrm{Cmol}$ ) of electron donor largely due to it being a three-carbon metabolite as opposed to the other compatible solutes which are five or six carbon metabolites. Hydroxyectoine synthesis had the largest requirements for electron donor and acceptor of the six considered compatible solutes.

The availability of oxygen strongly influenced the electron donor requirements to synthesize each compatible solute (Table 2). Transitioning from oxygen sufficiency to anoxic conditions with no external electron acceptors, the synthesis of ectoine required $\sim$ twofold more glucose. The absence of oxygen prevented the synthesis of all six compatible solutes based on citrate catabolism; the $\mathrm{BC} 1$ genome does not possess genes for citrate fermentation (Bott 1997).

The substrate requirements to synthesize each compatible solute were converted into ATP opportunity costs to create a comparative basis across conditions and molecules. The ATP opportunity cost calculated how much ATP could have been synthesized from the substrate if it had been used for energy production rather than compatible solute production. The opportunity costs for each of the six compatible solutes produced from either citrate or glucose and in the presence or absence of oxygen can be found in Table 3.

Counterintuitively, the ATP opportunity costs for anoxic synthesis of the six compatible solutes from glucose were lower than the aerobic ATP opportunity costs, illustrating the different relative value of substrate carbon as an energy source and as an anabolic building block. For example, synthesizing ectoine optimally from glucose required 7.1 Cmol glucose aerobically, but required $13.5 \mathrm{Cmol}$ glucose under anoxic conditions with no external electron acceptor. However in terms of ATP opportunity costs, the aerobic synthesis represented a larger number of ATP (23.7 ATP aerobically vs. 6.75 ATP anaerobically). Aerobically, each Cmol of glucose can produce up to $3.33 \mathrm{~mol}$ of ATP, but in the absence of oxygen, the same Cmol of glucose can only produce $0.5 \mathrm{~mol}$ of ATP (Table 3 ).

The metabolic costs to synthesize compatible solutes were also analyzed as a function of anabolic resource availability (e.g. nitrogen source). For these analyses, the number of enzymatic steps per EFM was used to quantify 

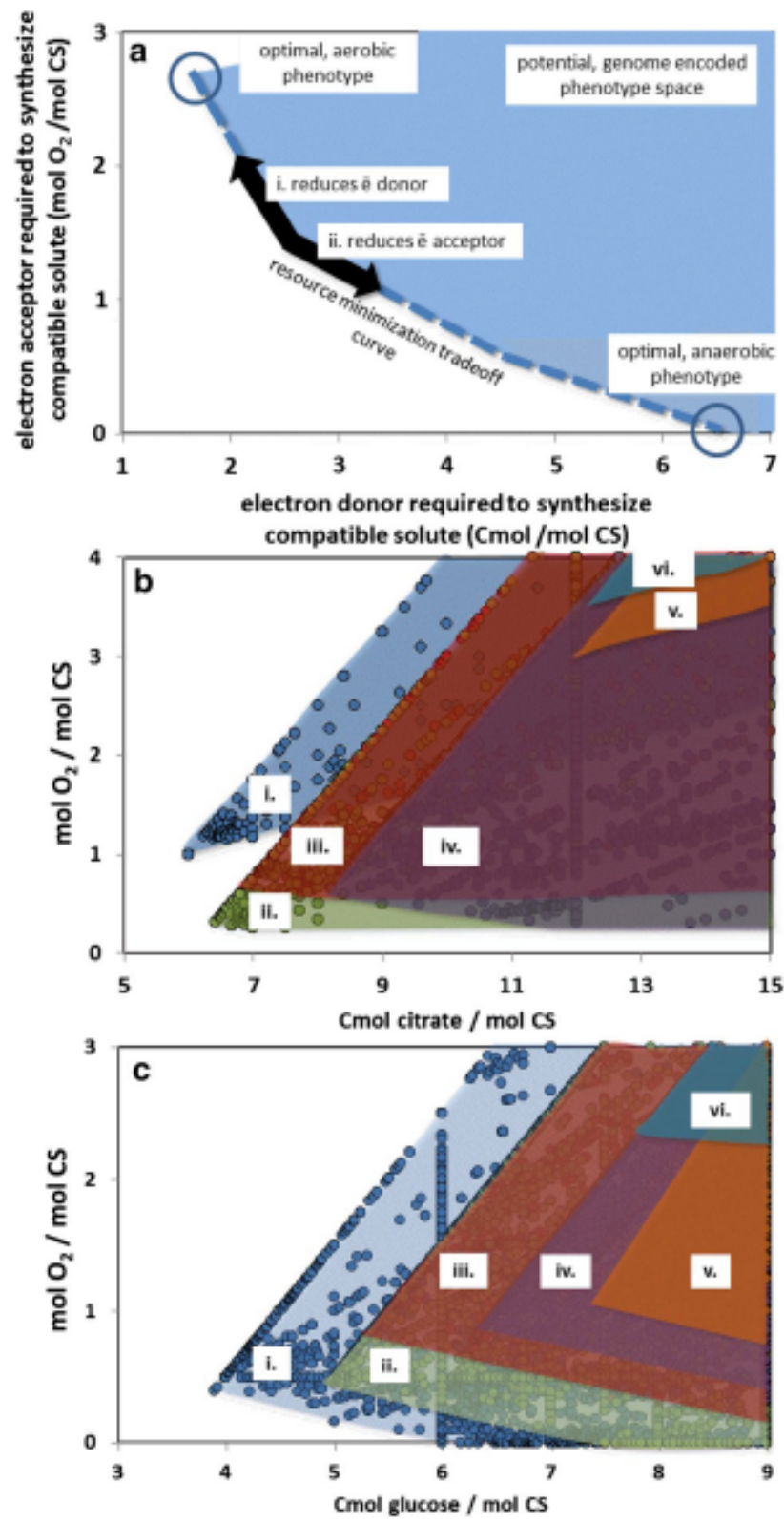

Fig. 5 In silico systems biology analysis of cellular economy of compatible solute synthesis in Halomonas BCl. a Diagram illustrating application of resource tradeoff theory for the analysis of metabolic networks. $x$ - and $y$-coordinates represent the cost to synthesize compatible solute (CS) in terms of the two resources (electron donor and electron acceptor). Dashed line segments identify the optimal tradeoff curve where the cost to synthesize the compatible solute is minimized in terms of the two resources. When $\mathrm{mol} \mathrm{O}_{2} / \mathrm{mol} \mathrm{CS}=0$, the culture is growing anaerobically (no external electron acceptor). b In silico resource tradeoff analysis of compatible solute synthesis using citrate as the electron donor (1. glycerol, 2. glutamate, 3. glutamine, 4. proline, 5. ectoine, 6. hydroxyectoine). $\mathrm{c}$ In silico resource tradeoff analysis of compatible solute synthesis using glucose as the electron donor (1. glycerol, 2. glutamate, 3. glutamine, 4. proline, 5. ectoine, 6. hydroxyectoine) the relative anabolic resource requirement for utilizing the EFM. Briefly, as anabolic resource availability decreased, the robust central metabolism can shift from high ATPyielding and high resource investment strategies to lower ATP-yielding, but also lower investment requiring strategies (Folsom et al. 2014). The low resource investment strategies result in the secretion of reduced carbon byproducts analogous to a fermentation metabolism with lactic acid being a common byproduct. See supplemental material for investment analysis for all six compatible solutes and ATP.

\section{Discussion}

Life under high salt and alkaline stress requires competitive physiological strategies. In a previous report (Oren 1999), the ATP requirements for synthesizing compatible solutes from glucose were calculated using nonspecific parameters and assuming oxygen sufficiency. The current work further develops this analysis through the use of an in silico metabolism model using molecular-level, isolate-specific data and through considering environmental stresses like oxygen availability. The compatible solute ATP costs calculated here differ from the previous study; the ATP opportunity costs are lower (Oren 1999). For example, aerobic synthesis of glycerol is calculated here to require $3.9 \mathrm{Cmol}$ of glucose which has an ATP opportunity cost of 13 ATP per glycerol (Table 3); the previous report calculated an ATP equivalent cost of approximately 22 ATP per glycerol. The difference is due primarily to assumptions regarding electron transport chain $\mathrm{H}^{+}$translocation efficiency and the number of $\mathrm{H}^{+}$required per ATP phosphodiester bond formed; the present study uses currently accepted values (Petersen et al. 2012; Steigmiller et al. 2008).

The use of ectoine as a compatible solute is widespread among halophiles despite it being more costly to synthesize than other compatible solutes including glycerol and some amino acids (Fig. 5). The substantial differences in resource requirements suggest there are functional benefits to ectoine that offset the higher nutrient requirements. Glycerol is relatively inexpensive to produce, but it is highly soluble in biological membranes making it a poorly retained compatible solute (Oren 1999). Amino acids are also relatively inexpensive to synthesize as compared to ectoine, but they are essential for cellular growth. During rapid salt dilution events like rain, halophiles often need to quickly reduce the cytosolic osmotic pressure. A common mechanism is to secrete the compatible solutes in a process known as the Birch Effect (Halverson et al. 2000; 
Table 3 Summary of compatible solute (CS) synthesis costs for Halomonas BC1 expressed as ATP opportunity costs

\begin{tabular}{lllll}
\hline Compatible solute & $\begin{array}{l}\text { ATP opportunity cost: citrate, } \\
\text { oxic }\end{array}$ & $\begin{array}{l}\text { ATP opportunity cost: citrate, } \\
\text { anoxic }\end{array}$ & $\begin{array}{l}\text { ATP opportunity cost: } \\
\text { glucose, oxic }\end{array}$ & $\begin{array}{l}\text { ATP opportunity cost: } \\
\text { glucose, anoxic }\end{array}$ \\
\hline Ectoine & 29.3 & Not possible & 23.7 & 6.8 \\
Glycerol & 14.6 & Not possible & 13.0 & 3 \\
Hydroxyectoine & 29.3 & Not possible & 26.1 & Not possible \\
L-Glutamate & 15.6 & Not possible & 16.6 & 3.8 \\
L-Glutamine & 16.7 & Not possible & 17.6 & 4.8 \\
L-Proline & 3.4 & Not possible & 21.0 & 11.8 \\
ATP & $\mathbf{1 4 . 5}$ & Not possible & $\mathbf{2 0}$ & $\mathbf{3}$ \\
\hline
\end{tabular}

The ATP opportunity costs quantified how many ATP could be synthesized if the substrate carbon [citrate (italics cells), or glucose] was used for energy synthesis rather than CS synthesis. The cost calculations used the optimal oxic and optimal anoxic yields for ATP synthesis from either citrate or glucose

a The bold ATP row documents the number of ATP that can be synthesized from either citrate or glucose, note this is per mole substrate not Cmole substrate. See supplemental material for more details

Kieft et al. 1987). Secreting amino acids would hamper or prevent cellular growth during salt dilution events. Ectoine, which has good cellular retention properties unlike glycerol, can be secreted during salt dilution events without compromising the ability of the cell to grow (unlike amino acids), perhaps justifying its resource intensive synthesis. Acquiring compatible solutes from the environment could reduce host osmotolerance-associated resources costs. The compatible solute glycine betaine is rarely synthesized de novo in bacteria; instead, it is often acquired from plantderived materials in the environment. $\mathrm{BC} 1$ acquired glycine betaine from the TSB medium rather than synthesizing ectoine (Fig. 3). The $\mathrm{BC} 1$ genome-encoded glycine betaine $\mathrm{ABC}$ transporter would require the equivalent of one ATP per compatible solute molecule acquired (aerobically and anaerobically), a substantially smaller cost than de novo synthesis of ectoine ( 7-29 ATP, Table 3).

Increasing salt concentration stresses cells in multiple ways. There is a need to pump more ions out of the cell, which is often an active process leading to higher cellular maintenance energy requirements, and the cell would need to synthesize more compatible solutes, which is another costly process. Additionally, increased salt concentrations lower the solubility of oxygen reducing its availability for energy metabolism (Pade et al. 2013; Sherwood et al. 1991). Increasing the salinity of an aqueous solution from $2.5(\mathrm{w} / \mathrm{v})$ to $10 \%(\mathrm{w} / \mathrm{v})$ at $30^{\circ} \mathrm{C}$ lowers oxygen solubility from $\sim 7$ to $4 \mathrm{mg} / \mathrm{L}$ (Sherwood et al. 1991). This would decrease oxygen flux to a cell $43 \%$ under transport-limited conditions. Using the in silico ATP tradeoff curve in the supplemental material, a $43 \%$ decrease in oxygen availability would translate into a $\sim 3$ fold increase in glucose consumption just to maintain the same ATP synthesis rate. If the higher salinity necessitated a larger ATP flux for cellular maintenance purposes, which is likely the case, the glucose flux increase would be even larger.

\section{Conclusions}

Haloalkaliphiles are an important class of microorganisms persisting in challenging ecological niches such as soda lakes, marine environments, hot springs, medical wounds, and industrial brine solutions. Decoding the basis of their metabolism under harsh conditions provides ecological knowledge fundamental to understanding their competitive strategies, as well as provides a rational template for controlling these organisms for bioprocess applications. Two Halomonas isolates $\mathrm{BC} 1$ and $\mathrm{BC} 2$ were purified from industrial brine solutions. The sequenced genomes revealed they were closely related to each other and to Halomonas meridiana and Halomonas stevensii. A physiological analysis of optimal salinity and $\mathrm{pH}$ levels revealed a remarkable tolerance to varying conditions and classified the isolates as moderate halophiles and alkaliphiles. An in silico metabolic model of isolate $\mathrm{BC} 1$ was constructed and used to study acclimation strategies to culturing stresses. This research makes substantial functional and theoretical contributions to our current understanding of Halomonas acclimations to salinity and $\mathrm{pH}$ stresses, results that are relevant to both general and applied microbiological research.

Acknowledgments The authors are grateful for the financial assistance of Procter and Gamble, Inc. and appreciate the contributions of Adam Kennedy and colleagues at Metabolon, Inc. The authors would also like to thank Ryan Jennings, Ashley Beck and Kristopher Hunt for critical reading of the manuscript and 16S rRNA analysis.

\section{References}

Aston JE, Peyton BM (2007) Response of Halomonas campisalis to saline stress: changes in growth kinetics, compatible solute production and membrane phospholipid fatty acid composition. FEMS Microbiol Lett 274:196-203 
Ates O, Oner ET, Arga KY (2011) Genome-scale reconstruction of metabolic network for a halophilic extremophile, Chromohalobacter salexigens DSM 3043. BMC Syst Biol 5:12

Beck A, Hunt KA, Bernstein HC, Carlson RP (2016) Interpreting and designing microbial communities for bioprocess applications, from components to interactions to emergent properties. Biotechnol Biofuel Prod Optim 1:407-432

Benjamini Y, Hochberg Y (1995) Controlling the false discovery rate: a practical and powerful approach to multiple testing. J R Stat Soc B Methodol 57:289-300

Biegel E, Schmidt S, González JM, Muller V (2011) Biochemistry, evolution and physiological function of the Rnf complex, a novel ion-motive electron transport complex in prokaryotes. Cell Mol Life Sci 68:613-634

Booth IR (1985) Regulation of cytoplasmic $\mathrm{pH}$ in bacteria. Microbiol Rev 49:359-378

Bott M (1997) Anaerobic citrate metabolism and its regulation in enterobacteria. Arch Microbiol 167:78-88

Brown AD (1976) Microbial water stress. Bacteriol Rev 40:803-846

Bursy J, Pierik AJ, Pica N, Bremer E (2007) Osmotically induced synthesis of the compatible solute hydroxyectoine is mediated by an evolutionarily conserved ectoine hydroxylase. J Biol Chem 282:31147-31155

Carlson RP (2007) Metabolic systems cost-benefit analysis for interpreting network structure and regulation. Bioinformatics 23:1258-1264

Carlson RP (2009) Decomposition of complex microbial behaviors into resource-based stress responses. Bioinformatics 25:90-97

Carlson RP, Taffs RL (2010) Molecular-level tradeoffs and metabolic adaptation to simultaneous stressors. Curr Opin Biotechnol $21: 670-676$

Carlson R, Wlaschin A, Srienc F (2005) Kinetic studies and biochemical pathway analysis of anaerobic poly-(R)-3-hydroxybutyric acid synthesis in Escherichia coli. Appl Environ Microbiol 71:713-720

Delcher AL, Bratke KA, Powers EC, Salzberg SL (2007) Identifying bacterial genes and endosymbiont DNA with Glimmer. Bioinformatics 23:673-679

Dimroth P, Jockel P, Schmid M (2001) Coupling mechanism of the oxaloacetate decarboxylase $\mathrm{Na}^{+}$pump. BBA Bioenerg 1505:1-14

Evans AM, DeHaven CD, Barrett T, Mitchell M, Milgram E (2009) Integrated, nontargeted ultrahigh performance liquid chromatography/electrospray ionization tandem mass spectrometry platform for the identification and relative quantification of the small-molecule complement of biological systems. Anal Chem 81:6656-6667

Folsom JP, Parker AE, Carlson RP (2014) Physiological and proteomic analysis of Escherichia coli iron-limited chemostat growth. J Bacteriol 196:2748-2761

Giordano A, Vella FM, Romano I, Gambacorta A (2007) Structural elucidation of a novel phosphoglycolipid isolated from six species of Halomonas. J Lipid Res 48:1825-1831

Goto T, Matsuno T, Hishinuma-Narisawa M, Yamazaki K, Matsuyama $\mathrm{H}$, Inoue $\mathrm{N}$, Yumoto I (2005) Cytochrome $\mathrm{c}$ and bioenergetic hypothetical model for alkaliphilic Bacillus spp. J Biosci Bioeng 100:365-379

Grammann K, Volke A, Kunte HJ (2002) New type of osmoregulated solute transporter identified in halophilic members of the bacteria domain: TRAP transporter TeaABC mediates uptake of ectoine and hydroxyectoine in Halomonas elongata DSM 2581T. J Bacteriol 184:3078-3085

Grant WD (2004) Life at low water activity. Philos Trans R Soc B 359:1249-1266

Guzman H, Van-Thuoc D, Martin J, Hatti-Kaul R, Quillaguaman $\mathbf{J}$ (2009) A process for the production of ectoine and poly(3-hydroxybutyrate) by Halomonas boliviensis. Appl Microbiol Biotechnol 84:1069-1077

Halverson LJ, Jones TM, Firestone MK (2000) Release of intracellular solutes by four soil bacteria exposed to dilution stress. Soil Sci Soc Am J 64:1630-1637

Horikoshi K (1999) Alkaliphiles: some applications of their products for biotechnology. Microbiol Mol Biol Rev 63:735-750

Kempf B, Bremer E (1998) Uptake and synthesis of compatible solutes as microbial stress responses to high-osmolality environments. Arch Microbiol 170:319-330

Kieft TL, Soroker E, Firestone MK (1987) Microbial biomass response to a rapid increase in water potential when dry soil is wetted. Soil Biol Biochem 19:119-126

Klamt S, Saez-Rodriguez J, Gilles ED (2007) Structural and functional analysis of cellular networks with CellNetAnalyzer. BMC Syst Biol 1:2

Krulwich TA, Ito M, Gilmour R, Sturr MG, Guffanti AA, Hicks DB (1996) Energetic problems of extremely alkaliphilic aerobes. BBA Bioenerg 1275:21-26

Kuhlmann SI, Terwisscha van Scheltinga AC, Bienert R, Kunte HJ, Ziegler C (2008) $1.55 \AA$ structure of the ectoine binding protein TeaA of the osmoregulated TRAP-transporter TeaABC from Halomonas elongata. Biochemistry 47:9475-9485

Kushner DJ (1978) Life in high salt and solute concentrations: halophilic bacteria. In: Kushner DJ (ed) Microbial life in extreme environments. Academic Press, London, pp 317-368

Kushner DJ, Kamekura M (1988) Physiology of halophilic eubacteria. Halophilic Bact 1:109-140

Mothes G, Schubert T, Harms H, Maskow T (2008) Biotechnological coproduction of compatible solutes and polyhydroxyalkanoates using the genus Halomonas. Eng Life Sci 8:658-662

Neidhardt FC, Ingraham JL, Schaechter M (1990) Physiology of the bacterial cell: a molecular approach. Sinauer Associates, Sunderland, MA

Oren A (1999) Bioenergetic aspects of halophilism. Microbiol Mol Biol Rev 63:334-348

Oren A (2008) Microbial life at high salt concentrations: phylogenetic and metabolic diversity. Saline Syst $4: 2$

Oren A (2011) Thermodynamic limits to microbial life at high salt concentrations. Environ Microbiol 13:1908-1923

Pade N, Köcher S, Roessler M, Hănelt I, Muller V (2013) Bioenergetics of the moderately halophilic bacterium Halobacillus halophilus: composition and regulation of the respiratory chain. Appl Environ Microbiol 79:3839-3846

Pastor JM, Bernal V, Salvador M, Argandona M, Vargas C, Csonka L, Sevilla A, Iborra JL, Nieto JJ, Canovas M (2013) Role of central metabolism in the osmoadaptation of the halophilic bacterium Chromohalobacter salexigens. J Biol Chem 288:17769-17781

Petersen J, Forster K, Turina P, Graber P (2012) Comparison of the $\mathrm{H}^{+}$/ATP ratios of the $\mathrm{H}^{+}$-ATP synthases from yeast and from chloroplast. PNAS 109:11150-11155

Roberts MF (2005) Organic compatible solutes of halotolerant and halophilic microorganisms. Saline Syst 1:5

Schuster S, Hilgetag C (1994) On elementary flux modes in biochemical reaction systems at steady state. J Biol Syst 2:165-182

Schuster R, Schuster S (1993) Refined algorithm and computer program for calculating all non-negative fluxes admissible in steady states of biochemical reaction systems with or without some flux rates fixed. Bioinformatics 9:79-85

Schuster S, Fell DA, Dandekar T (2000) A general definition of metabolic pathways useful for systematic organization and analysis of complex metabolic networks. Nat Biotechnol 18:326-332

Schwibbert K, Marin-Sanguino A, Bagyan I, Heidrich G, Lentzen G, Seitz H, Rampp M, Schuster SC, Klenk HP, Pfeiffer F, Oesterhelt D, Kunte HJ (2011) A blueprint of ectoine metabolism from 
the genome of the industrial producer Halomonas elongata DSM 2581. Environ Microbiol 13:1973-1994

Sherwood JE, Stagnitti F, Kokkinn MJ, Williams WD (1991) Dissolved-oxygen concentrations in hypersaline waters. Limnol Oceanogr 36:235-250

Smyth GK (2004) Linear models and empirical Bayes methods for assessing differential expression in microarray experiments. Stat Appl Genet Mol Biol 3:3

Smyth GK (2005) Limma: linear models for microarray data. Bioinformatics and computational biology solutions using $\mathrm{R}$ and bioconductor. Springer, New York, pp 397-420

Steigmiller S, Turina P, Graeber P (2008) The thermodynamic $\mathrm{H}^{+}$/ ATP ratios of the $\mathrm{H}^{+}$-ATPsynthases from chloroplasts and Escherichia coli. PNAS 105:3745-3750

Steuber J, Schmid C, Rufibach M, Dimroth P (2000) $\mathrm{Na}^{+}$translocation by complex I (NADH:quinone oxidoreductase) of Escherichia coli. Mol Microbiol 35:428-434
Tamura K, Stecher G, Peterson D, Filipski A, Kumar S (2013) MEGA6: molecular evolutionary genetics analysis version 6.0. Mol Biol Evol 30:2725-2729

Thompson JD, Higgins DG, Gibson TJ (1994) CLUSTAL W: improving the sensitivity of progressive multiple sequence alignment through sequence weighting, position-specific gap penalties and weight matrix choice. Nucl Acids Res 22:4673-4680

Trinh CT, Wlaschin A, Srienc F (2009) Elementary mode analysis: a useful metabolic pathway analysis tool for characterizing cellular metabolism. Appl Microbiol Biotechnol 81:813-826

Ventosa A, Nieto JJ, Oren A (1998) Biology of moderately halophilic aerobic bacteria. Microbiol Mol Biol Rev 62:504-544

Verkhovsky MI, Bogachev AV (2010) Sodium-translocating NADH: quinone oxidoreductase as a redox-driven ion pump. BBA 1797:738-746 